\title{
BMJ Open Association of low birth weight with cardiometabolic diseases in Swedish twins: a population-based cohort study
}

\author{
Xuerui Li, ${ }^{1,2,3}$ Rongrong Yang, ${ }^{4}$ Wenzhe Yang, ${ }^{1,2,3}$ Hui Xu, ${ }^{5}$ Ruixue Song, ${ }^{1,2,3}$ \\ Xiuying Qi, ${ }^{1,2,3}$ Weili Xu (i) ${ }^{1,2,3,6}$
}

To cite: Li X, Yang R, Yang W, et al. Association of low birth weight with cardiometabolic diseases in Swedish twins: a population-based cohort study. BMJ Open 2021;11:e048030. doi:10.1136/ bmjopen-2020-048030

- Prepublication history and additional supplemental material for this paper are available online. To view these files, please visit the journal online (http://dx.doi.org/10.1136/ bmjopen-2020-048030).

Received 23 December 2020 Accepted 10 June 2021

\section{Check for updates}

(C) Author(s) (or their employer(s)) 2021. Re-use permitted under CC BY. Published by BMJ.

For numbered affiliations see end of article.

\section{Correspondence to} DrWeili Xu; weili.xu@ki.se and Dr Xiuying Qi;

qixiuying@tmu.edu.cn

\section{ABSTRACT}

Objectives To examine the association between low birth weight (LBW) and cardiometabolic diseases (CMDs, including heart disease, stroke and type 2 diabetes mellitus) in adulthood, and to explore whether genetic, early-life environmental and healthy lifestyle factors play a role in this association.

Design A population-based twin study.

Setting Twins from the Swedish Twin Registry who were born in 1958 or earlier participated in the Screening Across the Lifespan Twin (SALT) study for a full-scale screening during 1998-2002 and were followed up until 2014. Participants 19779 twin individuals in Sweden with birthweight data available (mean age: 55.45 years). Primary and secondary outcome measures CMDs were assessed based on self-reported medical records, medication use and records from the National Patient Registry. A lifestyle index encompassing smoking status, alcohol consumption, exercise levels and Body Mass Index was derived from the SALT survey and categorised as unfavourable, intermediate or favourable. Data were analysed using generalised estimating equation (GEE) models and conditional logistic regression models. Results Of all participants, 3998 (20.2\%) had LBW and $5335(27.0 \%)$ had incident CMDs (mean age at onset: $63.64 \pm 13.26$ years). In GEE models, the OR of any CMD was 1.39 (95\% Cl 1.27 to 1.52) for LBW. In conditional logistic regression models, the LBW-CMD association became non-significant $(\mathrm{OR}=1.21,95 \%$ $\mathrm{Cl} 0.94$ to 1.56). The difference in ORs from the two models was statistically significant $(p<0.001)$. In the joint effect analysis, the multiadjusted OR of CMDs was 3.47 (95\% Cl 2.72 to 4.43$)$ for participants with LBW plus an unfavourable lifestyle and 1.25 (95\% $\mathrm{Cl} 0.96$ to 1.62) for those with LBW plus a favourable lifestyle.

Conclusion LBW is associated with an increased risk of adult CMDs, and genetic and early-life environmental factors may account for this association. However, a favourable lifestyle profile may modify this risk.

\section{INTRODUCTION}

With population ageing has come an increase in the prevalence of chronic diseases, especially heart diseases (ie, coronary heart diseases and heart failure), stroke and type 2 diabetes mellitus (T2DM). ${ }^{1}$ According to the WHO, heart diseases and stroke, so-called

\section{Strengths and limitations of this study}

This study provides an extraordinary opportunity to explore the association between low birth weight (LBW) and cardiometabolic diseases (CMDs) by using a twin study design to control for some unmeasured confounders.

- The investigation into factors that might compensate for the risk effect of LBW on CMDs is unique.

- Birth weight was based on self-reports, and nondifferential misclassification among different birthweight groups could not be ruled out, possibly leading to an underestimation of the observed associations.

- Some prenatal factors (such as gestational age, maternal smoking during pregnancy or premature birth) could not be controlled for, as information on these factors was not available.

- Potential variations of lifestyle factors during the follow-up also could not be assessed.

cardiovascular disease (CVD), are the leading cause of disease burden and death worldwide. $^{2}{ }^{3}$ About 17.6 million deaths were attributed to CVD globally in $2016 .^{2}$ Meanwhile, there were 451 million adults living with diabetes worldwide in 2017 (90\% of whom had T2DM), and this number is projected to increase to 693 million by $2045 .{ }^{45}$ All of these co-occurring chronic diseases have been defined as cardiometabolic diseases (CMDs). ${ }^{67}$

Recently, beyond the effects of some traditional risk factors including age, smoking, drinking and Body Mass Index (BMI) on individual CMDs, the role of early-life experiences in the future development of chronic diseases have drawn special attention. ${ }^{8}$ Birth weight, an early-life indicator, ${ }^{9}$ is frequently used to explore the effects of early-life experiences on the risk of individual CMDs in adulthood. Several cohort studies have shown that low birth weight (LBW) is associated with an increased risk of coronary heart disease, ${ }^{10}$ stroke $^{11}$ and T2DM, ${ }^{12}{ }^{13}$ but with 
some inconsistent findings. ${ }^{14} 15$ Moreover, many studies have examined the relationship between birth weight and metabolic syndrome with inconsistent results, ${ }^{16-18}$ but no studies have investigated the association of LBW with the risk of CMDs.

CMDs are complex genetic and lifestyle-related disorders, ${ }^{19-21}$ and birth weight may also be affected by genetic factors and intrauterine environment. ${ }^{22}$ However, the role of the genetic and early-life environmental factors (another term for shared environmental factors), such as intrauterine environment and prenatal nutritional status, in the association between birth weight and CMDs remains unclear. Twin studies make it possible to minimise potential confounding effects of unmeasured genetic predisposition and shared early-life environment when comparisons are made between twins. ${ }^{23} 24$ Apart from genetic factors, some modifiable lifestyle factors such as not smoking, moderate alcohol consumption, engagement in physical activities and maintaining a healthy weight have been reported to be linked to a lower risk of CVD or T2DM. ${ }^{25}{ }^{26}$ However, previous population-based cohort studies have only shown that healthy lifestyle (such as active physical activity, not smoking, moderate alcohol consumption and $\mathrm{BMI}<25$ ) may reduce the risk effect of LBW on the development of diabetes. ${ }^{27} 28$ Questions remain regarding whether and to what extent healthy lifestyle may mitigate the risk of LBW on CMDs more widely.

In the present study, we aimed to (1) verify the relationship between LBW and risk of CMDs using populationbased Sweden twin data and (2) explore whether genetic, early-life environmental and healthy lifestyle factors play a role in this association.

\section{METHODS}

\section{Study population}

This prospective, nested case-control study included twins from the nationwide Swedish Twin Registry (STR), which started in the 1960s. ${ }^{29}$ From 1998 to 2002, all living twins born in 1958 or earlier were recruited to participate in the Screening Across the Lifespan Twin (SALT) study, a full-scale screening through a computer-assisted telephone interview. Of the 19940 twin individuals in the SALT study with birthweight data available, we excluded 53 individuals with birth weights that were outliers (extreme values; ie, birth weight of $\leq 300 \mathrm{~g}$ or $\geq 4520 \mathrm{~g}$ ) to minimise possible misclassification and 108 individuals with type 1 diabetes. Finally, 19779 individuals were included in the current study (online supplemental figure S1).

\section{Data collection}

Data on age, sex, educational attainment, marital status and zygosity status were collected through the SALT survey. ${ }^{29}$ Zygosity status was categorised as monozygotic, dizygotic or undetermined zygosity on the basis of selfreported information about childhood resemblance, which was validated against biological markers with 95\%-99\% accuracy. ${ }^{29}$ Education was dichotomised into
$<8 \mathrm{vs} \geq 8$ years according to the number of years of formal schooling attained. Marital status was classified into married/cohabitating vs single (including divorced or widows/widowers).

Information on medical conditions including heart disease, stroke, T2DM and hypertension was derived from the National Patient Registry (NPR), which covers all inpatient diagnoses in Sweden from the 1960s and outpatient (specialist clinic) diagnoses from 2001 to $2014 .^{30}$ Each medical record in the NPR included up to eight discharge diagnoses according to the International Classification of Disease (ICD) codes. The ICD, 7th Revision, was used through 1968, the 8th Revision from 1969 to 1986, the 9th Revision from 1987 to 1996 and the 10th revision from 1997 to the end of 2014.

\section{Assessment of birth weight}

Data on birth weight was collected based on self-reports from SALT or STR. Generally, LBW was defined as birth weight of $<2500 \mathrm{~g}$ in singletons. ${ }^{31}$ However, twins may experience a more unfavourable intrauterine environment, causing them to have a lower birth weight (on average $800 \mathrm{~g}$ ) than singletons. ${ }^{32}$ Thus, birth weight in the present study was categorised as $<2 \mathrm{~kg}$ (LBW), $2-3 \mathrm{~kg}$ (moderate birth weight (MBW)) or $>3 \mathrm{~kg}$ (high birth weight (HBW) $),{ }^{32}$ considering its distribution.

\section{Ascertainment of CMD}

In the current analysis, CMDs included heart disease (coronary heart disease and heart failure), stroke (ischaemic stroke and haemorrhagic stroke) and T2DM, all of which were diagnosed based on self-reported medical records, medication use and NPR data. The detailed ICD codes for each disease are shown in the online supplemental table S1.

CMD status was categorised as CMD-free and any CMD (ie, presence any of heart disease, stroke and/or T2DM). The any CMD group was further classified as only one CMD (heart disease, stroke or T2DM), any two CMDs (any two of the following: heart disease, stroke and T2DM), and three or more CMDs (heart disease, stroke and T2DM).

\section{Assessment of lifestyle-related factors}

Information on lifestyle factors (including smoking status, alcohol consumption, physical exercise and BMI) was obtained from the SALT survey. In detail, smoking status was dichotomised as non-smoking versus former/ current smoker. Alcohol consumption was categorised as no/mild drinking versus heavy drinking based on the survey question asking whether participants have ever drunk excessively over a period. Data on physical exercise were collected by a question on average exercise with seven response options: (1) 'almost never', (2) 'much less than average', (3) 'less than average', (4) 'average', (5) 'more than average', (6) 'much more than average' and (7) 'maximum, 33 and was dichotomised as 'inactive', including the first four groups (1-4) and 'active', 
Table 1 Characteristics of the study population $(N=19779)$ by birth weight

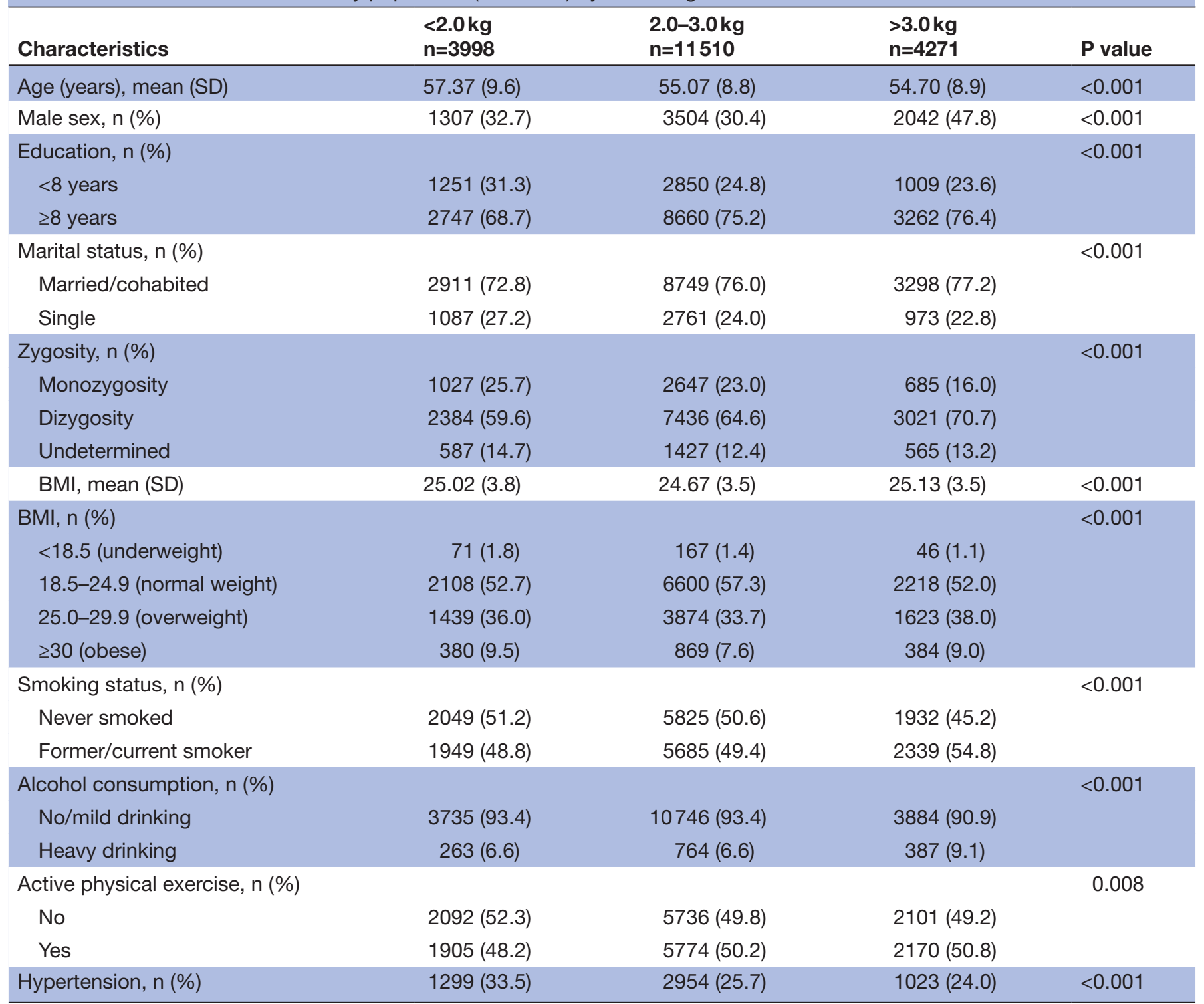

Data are presented as means \pm SD or number (\%).

BMI, body mass index.

including the last three groups (5-7). BMI in adulthood (mean age 55.45 \pm 9.05 ) was calculated as weight $(\mathrm{kg})$ divided by squared height $\left(\mathrm{m}^{2}\right)$ and classified as underweight $(<18.5)$, normal weight $(18.5-24.9)$, overweight (25.0-29.9) and obesity ( $\geq 30$ ) according to the WHO classification. Obesity was merged with overweight (hereafter overweight; ie, $\mathrm{BMI} \geq 25$ ), and underweight was merged with normal weight as non-overweight $(\mathrm{BMI}<25)$.

In the current study, on the basis of the data availability, the following four factors were considered as healthy lifestyle factors: (1) non-smoking, (2) no/mild alcohol consumption, (3) active physical exercise and (4) non-overweight in adulthood. ${ }^{34}$ The four factors were combined into a lifestyle index with a score ranging from 0 to 4 , with one point representing each factor. Participants were categorised according to their score of lifestyle index: (1) unfavourable (score: 0-1): participants who had no healthy lifestyle factors or only one, (2) intermediate (score: 2-3): those who had two or three healthy lifestyle factors, and (3) favourable (score: 4) those who had all the healthy lifestyle factors.

\section{Statistical analyses}

The characteristics of participants in different groups were compared using $\chi^{2}$ tests for categorical variables and one-way analysis of variance/Kruskal-Wallis $\mathrm{H}$ test for continuous variables. Missing values on education level $(\mathrm{n}=92)$, smoking status $(\mathrm{n}=77)$, alcohol consumption $(n=117)$, marital status $(n=2)$, physical exercise $(n=1179)$ and BMI $(n=290)$ were imputed using Rubin's rule for pooling estimates to obtain valid statistical inferences. ${ }^{24}$ 
Table 2 ORs and 95\% Cls of birth weight in relation to different subtypes of heart diseases, stroke and diabetes in adulthood: results from generalised estimating equation

\begin{tabular}{|c|c|c|c|}
\hline Single/combined CMDs & Cases (n) & OR $(95 \% \mathrm{Cl})^{*}$ & OR $(95 \% \mathrm{Cl}) \dagger$ \\
\hline \multicolumn{4}{|l|}{ Subtypes of heart disease } \\
\hline \multicolumn{4}{|l|}{$\mathrm{CHD}$} \\
\hline$<2$ & 622 & 1.33 (1.19 to 1.49$)$ & 1.27 (1.14 to 1.43$)$ \\
\hline $2-3$ & 1166 & Reference & Reference \\
\hline$>3$ & 497 & 1.07 (0.95 to 1.20$)$ & 1.08 (0.95 to 1.22$)$ \\
\hline \multicolumn{4}{|l|}{$\mathrm{HF}$} \\
\hline$<2$ & 214 & 1.36 (1.13 to 1.63$)$ & 1.27 (1.05 to 1.53$)$ \\
\hline $2-3$ & 356 & Reference & Reference \\
\hline$>3$ & 143 & 1.13 (0.93 to 1.39$)$ & 1.12 (0.91 to 1.38$)$ \\
\hline \multicolumn{4}{|l|}{ Subtypes of stroke } \\
\hline \multicolumn{4}{|l|}{ IS } \\
\hline$<2$ & 432 & 1.20 (1.06 to 1.36$)$ & 1.14 (1.01 to 1.30$)$ \\
\hline $2-3$ & 874 & Reference & Reference \\
\hline$>3$ & 352 & 1.10 (0.96 to 1.26$)$ & 1.12 (0.98 to 1.29$)$ \\
\hline \multicolumn{4}{|l|}{ HS } \\
\hline$<2$ & 74 & 1.14 (0.86 to 1.50$)$ & 1.09 (0.82 to 1.44$)$ \\
\hline $2-3$ & 162 & Reference & Reference \\
\hline$>3$ & 59 & 0.97 (0.72 to 1.32$)$ & 0.99 (0.73 to 1.34$)$ \\
\hline \multicolumn{4}{|l|}{ T2DM } \\
\hline$<2$ & 668 & 1.45 (1.30 to 1.61$)$ & 1.39 (1.24 to 1.55$)$ \\
\hline $2-3$ & 1219 & Reference & Reference \\
\hline$>3$ & 424 & 0.88 (0.78 to 0.99$)$ & $0.82(0.72$ to 0.93$)$ \\
\hline \multicolumn{4}{|c|}{ Any CMD (CHD, HF, IS and T2DM) } \\
\hline$<2$ & 1423 & 1.44 (1.32 to 1.57$)$ & $1.39(1.27$ to 1.52$)$ \\
\hline $2-3$ & 2797 & Reference & Reference \\
\hline$>3$ & 1115 & 1.06 (0.97 to 1.16$)$ & $1.05(0.96$ to 1.16$)$ \\
\hline
\end{tabular}

${ }^{*}$ Adjusted for age, sex and education.

†Adjusted for age, sex, education, Body Mass Index, smoking, alcohol consumption, marital status, physical exercise and hypertension. $\mathrm{CHD}$, coronary heart disease; CMD, cardiometabolic disease; HF, heart failure; HS, haemorrhagic stroke; IS, ischaemic stroke; T2DM, type 2 diabetes mellitus.

In our study, two analytical strategies were applied. First, generalised estimating equation (GEE) models were used for unmatched case-control analysis. GEE models are conceptually equivalent to logistic regression for the analysis of classic case-control design but control for the clustering of twins within a pair. Second, conditional logistic regression models were used for cotwin matched case-control analysis using a pair of twins that was discordant for the outcome. Cotwin matched design (especially in monozygotic twins) appeared more informative since cases and controls were comparable with respect to genetic background and early-life environmental factors such as intrauterine environment, prenatal and postnatal nutritional status, and childhood socioeconomic status. ${ }^{35}{ }^{36}$ In both GEE and conditional logistic regression, the ORs and 95\% CIs were estimated for the association between birth weight (reference:
MBW) and CMDs. Logistic regression was used to test the difference in ORs from GEE and conditional logistic regression models by examining the difference in the proportions of birth weight between unmatched controls and cotwin matched controls. ${ }^{36}$ If an OR for the observed association becomes strengthened or attenuated (or even disappears) in cotwin control analyses compared with that in the unmatched case-control analysis, and the difference in ORs from the two models is significant, then genetic and/or early-life environmental factors are likely to play a role in the association. ${ }^{2435}$ If the ORs are similar between the two models without a statistically significant difference, then the effect of genetic and/ or early-life environmental factors in the association can be neglected. ${ }^{23}{ }^{36}$ We hypothesised that LBW would be a significant risk factor for CMDs in a classical casecontrol analysis, but that the association between LBW 
Table 3 ORs and 95\% Cls for the association between LBW and adult CMDs in cotwin control analysis using CMDs discordant twin pairs: results from conditional logistic regression

\begin{tabular}{|c|c|c|c|c|c|c|}
\hline \multirow{2}{*}{ Cotwin control } & \multicolumn{6}{|c|}{ Cotwin with CMDs } \\
\hline & \multicolumn{2}{|c|}{$\begin{array}{l}\text { All zygosity twins* } \\
\text { ( } n=1293 \text { pairs) }\end{array}$} & \multicolumn{2}{|c|}{$\begin{array}{l}\text { Dizygotic only } \\
\text { ( } n=845 \text { pairs) }\end{array}$} & \multicolumn{2}{|c|}{$\begin{array}{l}\text { Monozygotic only } \\
\text { ( } n=290 \text { pairs) }\end{array}$} \\
\hline Non-LBW & 804 & 177 & 549 & 106 & 162 & 46 \\
\hline LBW & 153 & 159 & 90 & 100 & 45 & 37 \\
\hline
\end{tabular}

${ }^{*}$ Contain 158 pairs of undetermined zygosity twins.

†Adjusted for sex and education.

$\ddagger$ Adjusted for sex, education, body mass index, smoking, alcohol consumption, marital status, physical exercise and hypertension.

CMDs, cardiometabolic diseases; LBW, low birth weight.

and CMDs would be attenuated in the cotwin-matched analysis after controlling for genetic, maternal and environmental factors shared by twins. Logistic regression was used to test the difference in ORs from the GEE model and conditional logistic regression.

Considering information on lifestyle factors was obtained from the SALT questionnaire during 19982002, we excluded 1748 participants who developed CMDs before the SALT recruitment, and thus 18031 participants remained for the joint effect analysis. The combined effect of the LBW (no vs yes) and lifestyle index (unfavourable/intermediate/favourable) on the risk of CMDs was assessed by creating dummy variables based on the joint exposures to both factors. The presence of an additive interaction was examined by estimating relative excess risk due to interaction, the attributable proportion (AP) and the Synergy Index (S).

All the models were basic adjusted for age, sex and education, and further adjusted for smoking, alcohol consumption, marital status, physical exercise, BMI

Table 4 ORs and 95\% Cls of smoking, alcohol consumption, physical exercise and BMI related to cardiometabolic diseases from generalised estimating equation models

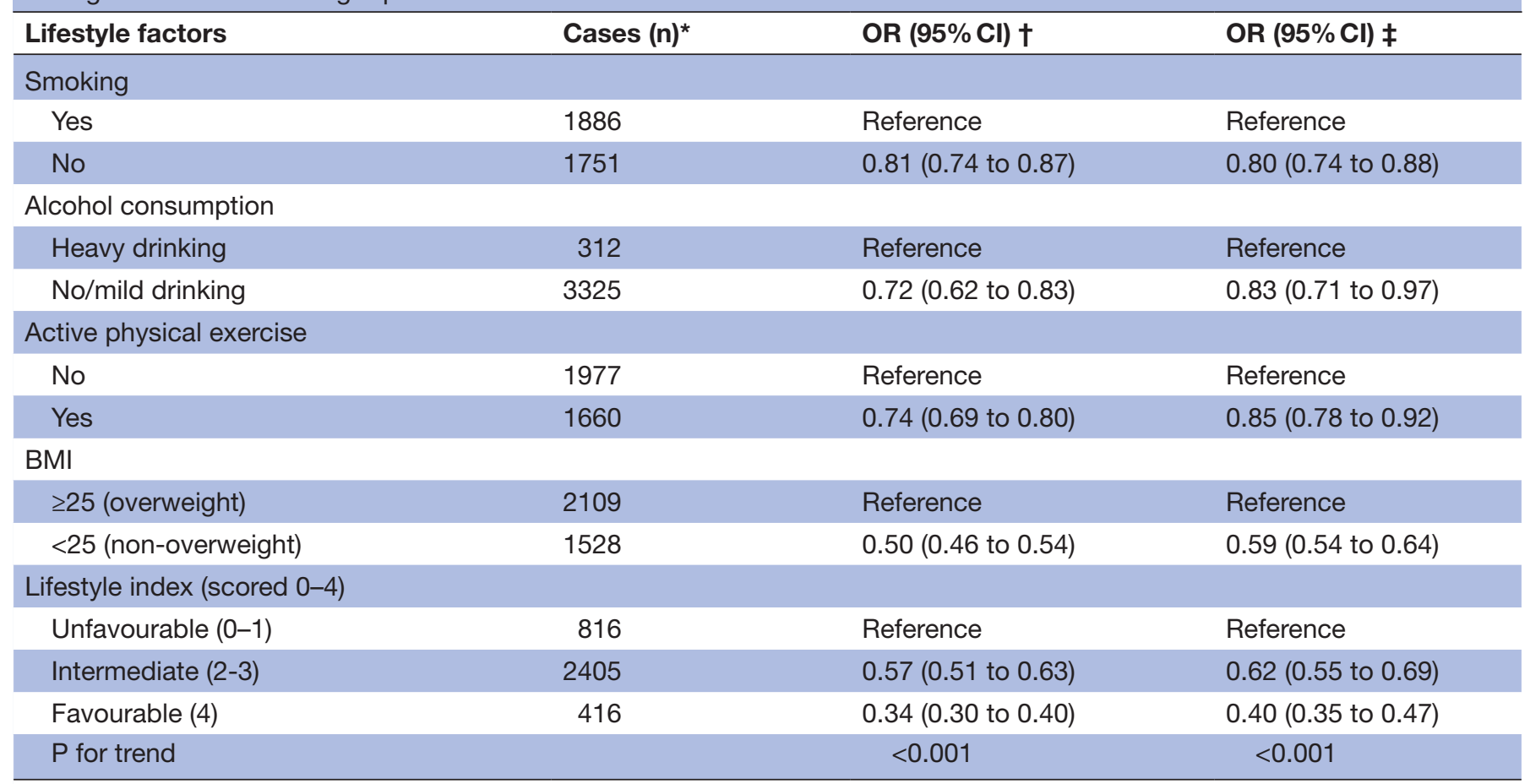

*1748 cases before Screening Across the Lifespan Twin study survey were excluded.

†Adjusted for age, sex and education.

$\ddagger$ Adjusted for age, sex, education, marital status, hypertension and birth weight, as well as BMI, smoking, alcohol consumption and active physical exercise, if applicable.

BMI, Body Mass Index. 
and hypertension. $\mathrm{P}$ values less than 0.05 were considered statistically significant. All statistical analyses were performed using SAS statistical software V.9.4 and IBM SPSS Statistics V.20.0.

\section{Patient and public involvement}

Patients and the public were not involved in the design, or conduct, or reporting of this study.

\section{RESULTS}

\section{Characteristics of the study population}

Among all participants $(\mathrm{n}=19779), 3998$ (20.2\%) had LBW. The average age at recruitment was $55.45( \pm 9.05)$ years. Compared with MBW individuals, those with LBW were more likely to be older, male, monozygotic twins, single, have lower education, have higher BMI, be physically inactive and have hypertension. Participants who had HBW were more likely to be male, dizygotic twins, smokers, heavy drinkers and have higher BMI (table 1).

\section{Association between birth weight and CMDs in unmatched case-}

\section{control analysis}

In the multiadjusted GEE model, compared with participants with MBW, those with LBW had a significantly higher risk of coronary heart disease, heart failure, ischaemic stroke and T2DM, which were further combined as CMDs $(\mathrm{n}=5335)$, as shown in table 2. LBW was associated with an increased risk of any CMD (OR 1.39, 95\% CI 1.27 to 1.52). However, HBW was not significantly associated with any CMD (OR 1.05, 95\% CI 0.96 to 1.16). Therefore, MBW and HBW were combined into non-LBW group as reference in the following analysis.

Compared with non-LBW, the OR for the association between LBW and any CMD was 1.37 (95\% CI 1.25 to 1.50). The multiadjusted ORs of LBW were $1.28(95 \%$ CI 1.17 to 1.41 ) for only one CMD, 1.48 (95\% CI 1.28 to 1.72 ) for any two CMDs and 1.82 (95\% CI 1.37 to 2.42) for three or more CMDs (reference: CMD-free), indicating the LBW-CMD risk became higher when multiple CMDs were co-occurring ( $p$ for trend $<0.001$ ) (online supplemental table S2). Further, the OR of the birth weight-CMD association was 0.84 (95\% CI 0.80 to 0.89 ) when birth weight was used as a continuous variable, suggesting a dose-dependent relationship between greater birth weight and lower CMD risk (online supplemental table S3).

\section{Association between LBW and CMDs in cotwin matched case- control analysis}

In the cotwin matched case-control analysis consisting of 845 dizygotic pairs and 290 monozygotic pairs, the association between LBW and any CMD was attenuated compared with the GEE model and became nonsignificant (OR 1.21, 95\% CI 0.94 to 1.56). The ORs for the associations were 1.34 (95\% CI 0.96 to 1.89 ) in dizygotic pairs and 1.07 (95\% CI 0.66 to 1.73 ) in monozygotic pairs (table 3 ).

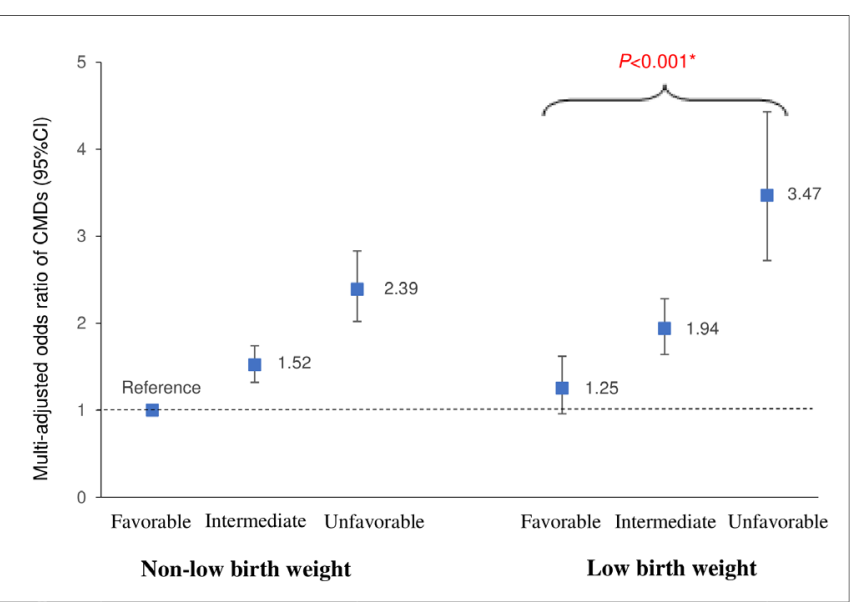

Figure 1 Joint effect of low birth weight (LBW) and lifestyle (smoking status, alcohol consumption, active physical exercise and Body Mass Index) on CMDs. Multiadjusted ORs $(95 \% \mathrm{Cl})$ of CMDs in relation to joint exposure of LBW and lifestyle from generalised estimating equation models (adjusted for age, sex, education, marital status and hypertension). ${ }^{*} P$ value of $<0.001$ refers to the difference in the risk of CMDs between participants with LBW who have a favourable lifestyle versus those with LBW who have an unfavourable lifestyle. CMDs, cardiometabolic diseases.

The difference in ORs from the GEE model versus conditional logistic model was statistically significant (OR $1.39,95 \%$ CI 1.21 to $1.59, \mathrm{p}<0.001$ ), which suggested that genetic and early-life environment factors might play an important role in LBW-CMD association.

\section{Association between lifestyle-related factors and CMDs}

In basic-adjusted and multiadjusted GEE models, not smoking, no/moderate alcohol drinking, active physical exercise and being non-overweight were individually related to a decreased risk of any CMD. When combined as a lifestyle index (unfavourable, intermediate and favourable), compared with an unfavourable lifestyle profile, an intermediate and a favourable lifestyle profile were significantly associated with a lower risk of any CMD, ORs were 0.62 (95\% CI 0.55 to 0.69$)$ and 0.40 (95\% CI 0.35 to 0.47 ), respectively (table 4 ).

\section{Joint effect of LBW and healthy lifestyle factors on CMD risk}

In the joint effect analysis, the multiadjusted ORs of any CMD were 1.25 (95\% CI 0.96 to 1.62) for participants with LBW plus a favourable lifestyle profile, 1.94 (95\% CI 1.64 to 2.28) for those with LBW plus an intermediate lifestyle profile, and 3.47 (95\% CI 2.72 to 4.43 ) for those with LBW plus an unfavourable lifestyle profile (reference: those with non-LBW plus a favourable lifestyle profile) (figure 1 and online supplemental table S4).

The additive interaction between the unfavourable lifestyle profile and LBW on CMDs was statistically significant (AP $0.199,95 \%$ CI 0.016 to $0.381, \mathrm{p}=0.03$; $\mathrm{S} 1.506,95 \%$ CI 1.001 to $2.267, \mathrm{p}<0.001$ ), indicating that if people with LBW have a favourable or intermediate lifestyle, the risk 
of LBW on CMDs can be reduced by $20 \%$ (online supplemental table S5).

\section{Supplementary analysis}

The results were not much altered compared with those from the initial analysis when we repeated the following analyses after: (1) stratifying by sex to address possible sex differences in the $\mathrm{CMDs}^{38}$ (online supplemental table S6), (2) additionally adjusting for survival status considering the association between LBW and mortality ${ }^{39}$ (online supplemental table S7), (3) excluding participants who developed CMDs before SALT recruitment $(\mathrm{n}=1748)$ (online supplemental table S8), (4) excluding participants with missing values for covariates $(n=1430)$ (online supplemental table S9), and (5) stratifying by twin birthweight concordance and discordance (online supplemental table S10).

\section{DISCUSSION}

In this large-scale, prospective, population-based nested case-control study of Swedish twins, we found that (1) LBW was associated with an increased risk of CMDs including coronary heart disease, heart failure, ischaemic stroke and T2DM in adulthood, and the risk became higher when multiple CMDs were co-occurring; (2) genetic background and early-life environmental factors appear to account for the LBW-CMD association; (3) a favourable lifestyle profile may modify the risk effect of LBW on CMDs.

Over the past two decades, the relationship between birth weight and T2DM ${ }^{121340}$ has been well documented. However, reports have been inconsistent regarding the association between birth weight and coronary heart disease. Three cohort studies have reported a relationship between LBW and the risk of coronary heart disease. ${ }^{101141}$ By contrast, Banci et al found that higher birth weight was associated with a higher risk of coronary heart disease. ${ }^{14}$ Another study showed there was no relationship between birth weight and coronary heart disease.$^{15}$ In addition, evidence on the relationship between LBW and heart failure or ischaemic stroke is sparse. To our knowledge, no studies have investigated the association of LBW with the risk of CMDs. In the present study, we found that LBW was associated with about $10 \%-40 \%$ increased risk of coronary heart disease, heart failure, ischaemic stroke (not haemorrhagic stroke) and T2DM. Further, we examined the relationship between birth weight and the risk of combined CMDs and found that individuals with LBW had an almost $40 \%$ higher risk of any CMD compared with those with non-LBW.

The potential contribution of genetic susceptibility and early-life environmental factors to the LBW-CMD association is still unclear. Previous twin cohort studies have shown that LBW is associated with an increased risk of CVD and T2DM when twins were considered as independent individuals. This association only held in outcomediscordant dizygotic twins but not in monozygotic twin pairs, suggesting that genetic mechanisms played a role in this association. ${ }^{13} 3242$ In the present study, we found that the LBW-CMD association became non-significant in both dizygotic and monozygotic twin pairs by using cotwin matched analyses. These results illustrated that early-life environmental factors could play an important role in the association between LBW and subsequent CMDs, along with genetic background.

Modifiable lifestyle factors (such as smoking, drinking, physical exercise and BMI) deserve to be studied in the context of the LBW-CMD association. To date, only a few studies have investigated the joint effect of LBW with lifestyle factors on T2DM. ${ }^{27} 2843$ One of the studies included 149794 participants from three large prospective cohorts and showed that LBW and unhealthy adulthood lifestyles encompassing smoking, non-moderate alcohol consumption, lower exercise intensity and $\mathrm{BMI} \geq 25$ were jointly related to an increased risk of T2DM. ${ }^{28}$ Another cohort study indicated that the risk of diabetes associated with LBW could be eliminated in those with a high physical activity level, ${ }^{27}$ and individuals predisposed to T2DM due to LBW could be protected from glucose intolerance by regular exercise ${ }^{43}$ However, no study has illustrated the joint effect of LBW and healthy lifestyle on subsequent CMDs. In the present study, we found that people with LBW and an intermediate or a favourable lifestyle profile (including not smoking, no/mild alcohol consumption, active physical exercise and being non-overweight) had a significantly lower risk of CMDs than those who had LBW and unfavourable lifestyle profile. To our knowledge, this is the first study to provide evidence that a healthy lifestyle might compensate for the risk effect of LBW on CMDs.

Several mechanisms may explain the relationship between LBW and the risk of CMDs. Twins have a unique and highly distinctive pattern of fetal growth. Although there is a higher rate of preterm birth among twins ${ }^{44}$ who may have lower birth weight compared with single births, a preterm fetus with LBW may have appropriate fetal growth. Actual growth restriction could occur when twins fail to adapt to an intrauterine environment. Foetal malnutrition or inappropriate growth in gestation may redirect scant energy supplies from muscle to vital tissues, causing permanent alterations in physiology, metabolism and structure. ${ }^{45}{ }^{46}$ Nevertheless, LBW alone could not fully capture the true growth level of the fetus, and monitoring the entire period of twin pregnancy is necessary to clarify the mechanism between LBW and CMDs in twins. Additionally, some genes (such as insulin class I allele or variants of mitochondrial DNA) have been associated with both birth weight loss and insulin resistance. ${ }^{47} 48$ All of these alterations could result in an increased risk of CVD and T2DM in adulthood. Moreover, a haplotype of the glucocorticoid receptor gene may modify the association between size at birth and glucose tolerance. ${ }^{49}$ However, maintaining a healthy lifestyle in adulthood may mitigate the risk of CMDs by improving insulin sensitivity and body composition, as well as controlling glycaemic, blood pressure and lipid profile. ${ }^{50}$ 


\section{Strengths and limitations}

Notable strengths of our study involve the large nationwide population-based twin cohort, which provided an extraordinary opportunity to explore the association between LBW and the risk of CMDs in adulthood by controlling for some unmeasured confounders, such as genetic background and early-life environmental factors. Furthermore, our investigation of potential compensatory factors against the LBW-CMD association is unique. Nevertheless, some limitations should be pointed out. First, hypertension was defined only based on self-reported data from the NPR, and subjects with undiagnosed hypertension might have been misclassified as hypertension-free. Thus, hypertension was not categorised as a CMD in the current study. Second, the assessment of birth weight was based on self-report so potential information bias could not be ruled out. However, such bias is more likely to be non-differential misclassification resulting in underestimation for the given associations. Third, data on gestational age and other prenatal factors (such as maternal smoking during pregnancy, premature birth or parental socioeconomic status) were not available and could not be fully controlled for. In addition, potential variations in lifestyle factors during follow-up could not be assessed. Fourth, diet could be partially taken into account, as it is closely associated with other lifestyle factors such as smoking, alcohol consumption, physical exercise and BMI. ${ }^{51}$ However, data on diet were not available in the SALT study. Finally, LBW in this study was defined as $<2.0 \mathrm{~kg}$ in twins. Caution is needed when generalising our findings to other populations.

\section{CONCLUSION}

This study provides evidence that LBW is associated with increased risk of CMDs including coronary heart disease, heart failure, ischaemic stroke and T2DM. The risk of CMDs related to LBW tends to increase with the number of co-occurring CMDs. Further, genetic and early-life environmental factors play an important role in the LBW-CMD association. However, a favourable lifestyle involving not smoking, no/mild alcohol consumption, active physical exercise and a BMI of $<25$ may compensate for the risk effect of LBW on CMDs. Our findings highlight the need for monitoring and controlling LBW for the prevention of CMDs, and the importance of maintaining a favourable lifestyle profile in people with LBW in adulthood to reduce the risk of CMDs.

\footnotetext{
Author affiliations

${ }^{1}$ Department of Epidemiology and Biostatistics, School of Public Health, Tianjin Medical University, Tianjin, China

${ }^{2}$ Tianjin Key Laboratory of Environment, Nutrition and Public Health, Tianjin, China

${ }^{3}$ Center for International Collaborative Research on Environment, Nutrition and Public Health, Tianjin, China

${ }^{4}$ Public Health Science and Engineering College, Tianjin University of Traditional Chinese Medicine, Tianjin, China

${ }^{5}$ Big Data and Engineering Research Center, Beijing Children's Hospital, Capital Medical University, National Center for Children's Health, Beijing, China
}

${ }^{6}$ Aging Research Center, Department of Neurobiology, Health Care Sciences and Society, Karolinska Institutet and Stockholm University, Stockholm, Sweden

Acknowledgements We thank the Swedish Twin Registry (STR) for access to the data, and we are grateful to all the twins who took part in the study, as well as the members of the survey teams. We are grateful to Professor Nancy L Pedersen for her great contribution to the design of the twin data collection. The STR is managed by Karolinska Institutet and receives funding through the Swedish Research Council (grant number 2017-00641).

Contributors WX conceptualised and designed the study and obtained funding for the study. XL conducted the literature search, analysed the data and wrote the first draft. XL, RY, WY, HX, RS, XQ and WX contributed to the discussion and interpretation of the results. WX and $X Q$ were involved in study supervision. All authors contributed to critical revision of the manuscript for important intellectual content and gave their final approval of the version to be published. XL and WX had full access to all the data in the study and take responsibility for the integrity of the data and the accuracy of the data analysis.

Funding This work was supported by grants from the Swedish Research Council (number 2017-00981), the National Natural Science Foundation of China (number 81771519), the Konung Gustaf V:s och Drottning Victorias Frimurare Foundation (number 2016-2020), Demensfonden, Strokefonden, Cornells Stiftelse and Alzheimerfonden (2018-2019). This project is part of CoSTREAM (www.costream. eu) and received funding from the European Union's Horizon 2020 research and innovation programme (grant agreement number 667 375). The funders had no role in the design and conduct of the study; collection, management, analysis and interpretation of the data; preparation, review or approval of the manuscript; and decision to submit the manuscript for publication.

Competing interests None declared.

Patient consent for publication Not required.

Ethics approval Informed consent was acquired from all participants. Data collection procedures were approved by the regional ethics committee at Karolinska Institutet, Stockholm, Sweden and the institutional review board of the University of Southern California, USA.

Provenance and peer review Not commissioned; externally peer reviewed.

Data availability statement Data are available upon reasonable request.

Supplemental material This content has been supplied by the author(s). It has not been vetted by BMJ Publishing Group Limited (BMJ) and may not have been peer-reviewed. Any opinions or recommendations discussed are solely those of the author(s) and are not endorsed by BMJ. BMJ disclaims all liability and responsibility arising from any reliance placed on the content. Where the content includes any translated material, BMJ does not warrant the accuracy and reliability of the translations (including but not limited to local regulations, clinical guidelines, terminology, drug names and drug dosages), and is not responsible for any error and/or omissions arising from translation and adaptation or otherwise.

Open access This is an open access article distributed in accordance with the Creative Commons Attribution 4.0 Unported (CC BY 4.0) license, which permits others to copy, redistribute, remix, transform and build upon this work for any purpose, provided the original work is properly cited, a link to the licence is given, and indication of whether changes were made. See: https://creativecommons.org/ licenses/by/4.0/.

ORCID iD

Weili Xu http://orcid.org/0000-0001-6140-2968

\section{REFERENCES}

1 Kivimäki M, Kuosma E, Ferrie JE, et al. Overweight, obesity, and risk of cardiometabolic multimorbidity: pooled analysis of individual-level data for 120813 adults from 16 cohort studies from the USA and Europe. Lancet Public Health 2017;2:e277-85.

2 Benjamin EJ, Muntner P, Alonso A, et al. Heart disease and stroke Statistics-2019 update: a report from the American heart association. Circulation 2019;139:e56-28.

3 GBD 2016 Disease and Injury Incidence and Prevalence Collaborators. Global, regional, and national incidence, prevalence, and years lived with disability for 328 diseases and injuries for 195 countries, 1990-2016: a systematic analysis for the global burden of disease study 2016. Lancet 2017;390:1211-59. 
4 Chatterjee S, Khunti K, Davies MJ. Type 2 diabetes. Lancet 2017;389:2239-51.

5 Cho NH, Shaw JE, Karuranga S, et al. IDF diabetes atlas: global estimates of diabetes prevalence for 2017 and projections for 2045. Diabetes Res Clin Pract 2018;138:271-81.

6 Wang Z, Marseglia A, Shang Y, et al. Leisure activity and social integration mitigate the risk of dementia related to cardiometabolic diseases: a population-based longitudinal study. Alzheimers Dement 2020;16:316-25.

7 Keenan T, Zhao W, Rasheed A, et al. Causal assessment of serum urate levels in cardiometabolic diseases through a Mendelian randomization study. J Am Coll Cardiol 2016;67:407-16.

8 Smith CJ, Ryckman KK, Barnabei VM, et al. The impact of birth weight on cardiovascular disease risk in the women's health Initiative. Nutr Metab Cardiovasc Dis 2016;26:239-45.

9 Lawani SO, Demerath EW, Lopez FL, et al. Birth weight and the risk of atrial fibrillation in whites and African Americans: the Atherosclerosis risk in communities (ARIC) study. BMC Cardiovasc Disord 2014;14:69.

10 Rich-Edwards JW, Kleinman K, Michels KB, et al. Longitudinal study of birth weight and adult body mass index in predicting risk of coronary heart disease and stroke in women. BMJ 2005;330:1115.

11 Lawlor DA, Ronalds G, Clark H, et al. Birth weight is inversely associated with incident coronary heart disease and stroke among individuals born in the 1950s: findings from the Aberdeen children of the 1950s prospective cohort study. Circulation 2005;112:1414-8.

12 Whincup PH, Kaye SJ, Owen CG, et al. Birth weight and risk of type 2 diabetes: a systematic review. JAMA 2008;300:2886-97.

13 Johansson S, lliadou A, Bergvall N, et al. The association between low birth weight and type 2 diabetes: contribution of genetic factors. Epidemiology 2008;19:659-65.

14 Banci M, Saccucci P, Dofcaci A, et al. Birth weight and coronary artery disease. The effect of gender and diabetes. Int J Biol Sci 2009;5:244-8.

15 Morley R, McCalman J, Carlin JB. Birthweight and coronary heart disease in a cohort born 1857-1900 in Melbourne, Australia. Int $J$ Epidemiol 2006;35:880-5.

16 Xiao X, Zhang Z-X, Li W-H, et al. Low birth weight is associated with components of the metabolic syndrome. Metabolism 2010;59:1282-6.

17 dos Santos Alves PdeJ, P T Henriques AC, Pinto LRM, et al. Endothelial and metabolic disorders in adolescence: low birth weight is not an isolated risk factor. $J$ Pediatr Endocrinol Metab 2015;28:407-13.

18 Briskiewicz BL, Barreto SM, do Amaral JF, et al. Early-life nutritional status and metabolic syndrome: gender-specific associations from a cross-sectional analysis of the Brazilian longitudinal study of adult health (ELSA-Brasil). Public Health Nutr 2018;21:1546-53.

19 Marenberg ME, Risch N, Berkman LF, et al. Genetic susceptibility to death from coronary heart disease in a study of twins. N Engl J Med 1994;330:1041-6.

20 Almgren P, Lehtovirta M, Isomaa B, et al. Heritability and familiality of type 2 diabetes and related quantitative traits in the Botnia study. Diabetologia 2011;54:2811-9.

21 Bak S, Gaist D, Sindrup SH, et al. Genetic liability in stroke: a longterm follow-up study of Danish twins. Stroke 2002;33:769-74.

22 Lunde A, Melve KK, Gjessing HK, et al. Genetic and environmental influences on birth weight, birth length, head circumference, and gestational age by use of population-based parent-offspring data. Am J Epidemiol 2007;165:734-41.

23 Xu W, Qiu C, Gatz M, et al. Mid- and late-life diabetes in relation to the risk of dementia: a population-based twin study. Diabetes 2009;58:71-7.

24 Yang R, Pedersen NL, Bao C, et al. Type 2 diabetes in midlife and risk of cerebrovascular disease in late life: a prospective nested case-control study in a nationwide Swedish twin cohort. Diabetologia 2019;62:1403-11.

25 Lv J, Yu C, Guo Y, et al. Adherence to Healthy Lifestyle and Cardiovascular Diseases in the Chinese Population. J Am Coll Cardiol 2017;69:1116-25.

26 Lv J, Yu C, Guo Y, et al. Adherence to a healthy lifestyle and the risk of type 2 diabetes in Chinese adults. Int J Epidemiol 2017;46:1410-20.
$27 \mathrm{Hu}$ C, Mu Y, Wan Q, et al. Association between birth weight and diabetes: role of body mass index and lifestyle in later life. J Diabetes 2020;12:10-20.

$28 \mathrm{Li}$ Y, Ley SH, Tobias DK, et al. Birth weight and later life adherence to unhealthy lifestyles in predicting type 2 diabetes: prospective cohort study. BMJ 2015;351:h3672.

29 Lichtenstein P, De Faire U, Floderus B, et al. The Swedish twin registry: a unique resource for clinical, epidemiological and genetic studies. J Intern Med 2002;252:184-205.

30 Patientregistret - Socialstyrelsen. Available: https://www. socialstyrelsen.se/statistik-och-data/register/alla-register/ patientregistret/ [Accessed 20 Oct 2020].

31 Xiao X, Zhang Z-X, Cohen HJ, et al. Evidence of a relationship between infant birth weight and later diabetes and impaired glucose regulation in a Chinese population. Diabetes Care 2008:31:483-7.

32 Hubinette A, Cnattingius S, Johansson ALV, et al. Birth weight and risk of angina pectoris: analysis in Swedish twins. Eur J Epidemiol 2002;18:539-44.

33 Carlsson S, Ahlbom A, Lichtenstein P, et al. Shared genetic influence of BMI, physical activity and type 2 diabetes: a twin study. Diabetologia 2013;56:1031-5.

34 Nyberg ST, Singh-Manoux A, Pentti J, et al. Association of healthy lifestyle with years lived without major chronic diseases. JAMA Intern Med 2020;180:760-8.

35 Kato K, Sullivan PF, Evengård B, et al. Premorbid predictors of chronic fatigue. Arch Gen Psychiatry 2006;63:1267-72.

$36 \mathrm{Xu}$ WL, Atti AR, Gatz M, et al. Midlife overweight and obesity increase late-life dementia risk: a population-based twin study. Neurology 2011;76:1568-74.

37 Bao C, Yang R, Pedersen NL, et al. Overweight in midlife and risk of cancer in late life: a nationwide Swedish twin study. Int $J$ Cancer 2019;144:2128-34.

38 Kuznetsova T. Sex differences in epidemiology of cardiac and vascular disease. Adv Exp Med Biol 2018;1065:61-70.

39 Hoy WE, Nicol JL. The Barker hypothesis confirmed: association of low birth weight with all-cause natural deaths in young adult life in a remote Australian Aboriginal community. J Dev Orig Health Dis 2019;10:55-62.

40 Kaijser M, Bonamy A-KE, Akre O, et al. Perinatal risk factors for diabetes in later life. Diabetes 2009;58:523-6.

41 Andersen LG, Angquist L, Eriksson JG, et al. Birth weight, childhood body mass index and risk of coronary heart disease in adults: combined historical cohort studies. PLoS One 2010;5:e14126.

42 Oberg S, Cnattingius S, Sandin S, et al. Birth weight predicts risk of cardiovascular disease within dizygotic but not monozygotic twin pairs: a large population-based co-twin-control study. Circulation 2011:123:2792-8.

43 Eriksson JG, Ylihärsilä H, Forsén T, et al. Exercise protects against glucose intolerance in individuals with a small body size at birth. Prev Med 2004;39:164-7.

44 Elliott JP, Istwan NB, Collins A, et al. Indicated and non-indicated preterm delivery in twin gestations: impact on neonatal outcome and cost. J Perinatol 2005;25:4-7.

45 Barker DJ. Fetal origins of coronary heart disease. BMJ 1995:311:171-4.

46 Poulsen P, Vaag A. Glucose and insulin metabolism in twins: influence of zygosity and birth weight. Twin Res 2001;4:350-5.

47 Dunger DB, Ong KKL, Huxtable SJ, et al. Association of the INS VNTR with size at birth. Nat Genet 1998;19:98-100.

48 Casteels K, Ong K, Phillips D. Mitochondrial 16189 variant, thinness at birth, and type-2 diabetes. Lancet 1999;353:1499-500.

49 Rautanen A, Eriksson JG, Kere J, et al. Associations of body size at birth with late-life cortisol concentrations and glucose tolerance are modified by haplotypes of the glucocorticoid receptor gene. $J$ Clin Endocrinol Metab 2006;91:4544-51.

50 Marwick TH, Hordern MD, Miller T, et al. Exercise training for type 2 diabetes mellitus: impact on cardiovascular risk: a scientific statement from the American Heart Association. Circulation 2009;119:3244-62.

51 Fransen HP, Boer JMA, Beulens JWJ, et al. Associations between lifestyle factors and an unhealthy diet. Eur J Public Health 2017;27:274-8 